12.1

\title{
Времяпролетная оптическая диагностика мощных импульсных ионных пучков
}

\author{
(C) В.А. Рыжков, Б.А. Нечаев, В.Н. Падалко
}

Исследовательская школа физики высокоэнергетических процессов, Томский политехнический университет, Томск, Россия

E-mail: ryzhkov@tpu.ru

Поступило в Редакцию 5 ноября 2019 г.

В окончательной редакции 16 января 2020 г.

Принято к публикации 20 января 2020 г.

Для контроля флюенсов ионов использована абляция тонкого слоя поверхностных загрязнений мишени, самовосстанавливающегося после каждого импульса мощного ионного пучка. С помощью времяпролетного оптического спектрометра измерены средние скорости самых легких компонентов абляционной плазмы, водорода и углерода, по их разности определен флюенс ионов.

Ключевые слова: загрязнение поверхности, вложение энергии, абляционная плазма, водород, углерод.

DOI: 10.21883/PJTF.2020.07.49222.18097

В работе [1] было показано, что слой поверхностных загрязнений твердой мишени, очищаемой мощным импульсным ионным пучком (МИП), частично восстанавливается в течение нескольких секунд после обработки. Активированная ионами поверхность адсорбирует водород, углерод и кислород из паров воды и масел остаточной атмосферы вакуумной камеры ускорителей. В [2] с помощью времяпролетной масс-спектрометрии было показано, что основной вклад в состав остаточного газа в камере циклотрона вносят молекулы используемого вакуумного масла (до $82 \mathrm{vol} . \%$ ) и молекулы воды (до 15-17 vol.\%), а в ионопроводе - молекулы воды (до 63 vol.\%). В работе [3] методом зонда Ленгмюра были исследованы времяпролетные характеристики абляционного факела, возникавшего при короткоимпульсном (15 ns) вложении энергии излучения лазера с длиной волны $532 \mathrm{~nm}$ в двухкомпонентную мишень $\mathrm{MnSi}$ в вакууме и в атмосфере аргона, по которым были получены функции распределения концентрации заряженных частиц по скорости разлета для различных значений давления буферного газа в вакуумной камере. В работе [4] измерялись времяразрешенные спектры эмиссии света, излучаемого распространяющимся факелом абляционной плазмы, генерированной мощным ионным пучком технологического ускорителя ТЕМП-2 в полиэтиленовой мишени, и было показано, что основные продукты расширяющегося факела представлены элементами матрицы: $\mathrm{H}, \mathrm{C}, \mathrm{C}_{2}$ и $\mathrm{CH}$.

Углеводородные загрязнения поверхности сублимируют при вложениях удельной энергии на уровне $0.2-0.3 \mathrm{~J} / \mathrm{mg}$, что достижимо для $300-400 \mathrm{keV}$ протонов уже при их флюенсах на уровне $10^{13}$ protons $/ \mathrm{cm}^{2}$ за импульс. При этом сублимированные продукты поверхностных загрязнений $(\mathrm{H}, \mathrm{C}, \mathrm{N}$ и $\mathrm{O})$ могут быть атомизированы и ионизированы настолько, чтобы образовать факел разряженной абляционной плазмы, в силу геометрии распространяющейся преимущественно по нормали от поверхности мишени. Наиболее легкие компоненты плазмы (водород и углерод), а также кислород должны распространяться на фронте факела, что позволяет осуществлять времяпролетную спектрометрию этих самых быстрых газовых компонентов и расчет по их относительным скоростям энергии $Q$, вложенной в единичную площадь мишени.

Без учета газодинамического ускорения граничного слоя плазмы и потерь энергии за время ее вложения ионами среднюю скорость $v_{i}$ компонентов абляционной плазмы (Н, С, О и др.) можно грубо определить их массами $M_{i}$ и температурой $T$ сразу после импульса:

$$
v_{i}=\sqrt{3 k T / M_{i}}
$$

где $k \approx 1.38 \cdot 10^{-23} \mathrm{~J} / \mathrm{K}$ - постоянная Больцмана.

Температуру можно выразить через вложенную энергию $Q$ или флюенс ионов $\Phi$ как

$$
T=Q / C_{p}=S_{a v} \Phi / C_{p},
$$

где $S_{a v}$ - средние тормозные потери энергии ионов в слое $\left[\mathrm{J} /\left(\mathrm{mg} \cdot \mathrm{cm}^{2}\right)\right]$, а $C_{p}-$ удельная теплоемкость углеводородов загрязнения при постоянном давлении.

Поскольку величину удельной теплоемкости слоя загрязнений определить точно невозможно, наилучшим выглядит относительный расчет вложенной энергии или флюенса ионов по разнице времен пролета базы длиной $L$ самыми быстрыми компонентами плазмы, водородной $\left(\tau_{\mathrm{H}}\right)$ и углеродной $\left(\tau_{\mathrm{C}}\right)$, когда коэффициент пропорциональности $K$ находят с помощью калибровки для каждой серии однотипных экспериментов:

$$
Q=S_{a v} \Phi=K L^{2} / \Delta \tau_{\mathrm{C}-\mathrm{H}}^{2} .
$$

За счет восстановления слоя поверхностных загрязнений в течение нескольких секунд становится возможным 
Contamination

layer

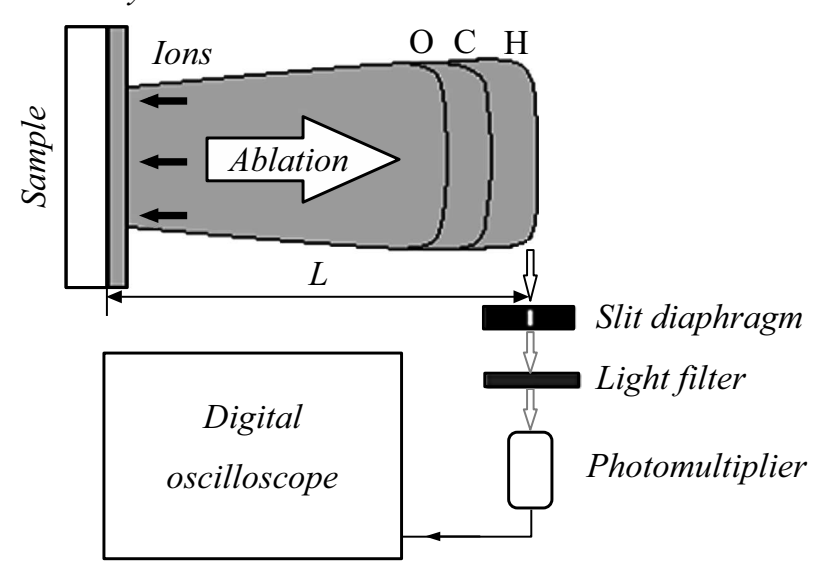

Pис. 1. Схема оптического времяпролетного спектрометра.

определять вложенную энергию $Q$ или флюенс ионов $\Phi$ сразу после каждого импульса МИП путем измерения относительных скоростей Н- и С- (и/или О-) компонентов. Крайне малая толщина слоя $(\ll 100 \mathrm{~nm})$ не влияет на скорости водородного и углеродного компонентов и, как следствие, на определение флюенса ионов и вложенной энергии. Состав слоя загрязнений практически не зависит от типа обрабатываемого материала, поэтому любой удобный для последующей калибровки материал может быть задействован во времяпролетной спектрометрии.

Задачей настоящей работы является проверка возможности диагностики мощных импульсных ионных пучков по времени пролета водородного и углеродного компонентов абляционной плазмы, генерированной в слое поверхностных загрязнений. Измерение скоростей распространения водорода и углерода абляционной плазмы было проведено с помощью оптической времяпролетной спектрометрии распространения светящегося фронта абляционного факела. На рис. 1 представлена схема оптического времяпролетного спектрометра, использованного для диагностики пучка технологического ускорителя ТЕМП-1 (Томский политехнический университет). Узкий слой фронта факела разряженной абляционной плазмы, образуемой слоем поверхностных загрязнений на титановой фольге, после каждого импульса ускорителя ТЕМП-1 просматривался через щелевую диафрагму $(1-2 \mathrm{~mm})$ и световые фильтры фотоэлектронным умножителем ФЭУ110, сопряженным с цифровым осциллографом Tektronix TDS224. Щелевая диафрагма отбирает фотоны, испускаемые компонентами абляционной плазмы на расстояниях $1-3 \mathrm{~cm}$ от мишени. Поскольку водород и углерод - самые быстрые компоненты просматриваемой абляционной плазмы, светофильтры выбирались серые, чтобы обеспечить максимально широкий диапазон пропускания света и снизить загрузку ФЭУ для самых первых импульсов серий, когда амплитуда сигнала предполагается в несколько раз большей, чем для остальных импульсов в среднем. Когда необходимо, селективными фильтрами может быть достигнуто дополнительное сепарирование аналитических сигналов избирательным поглощением фотонов мешающих компонентов абляционной плазмы.

На рис. 2 показаны времяпролетные спектры первого, второго, пятого и шестого импульсов одной серии, а в таблице приведены суммарные результаты по этим импульсам. Видно, что сигналы от водородного и углеродного компонентов разделяются достаточно просто, а при увеличении флюенса ионов на мишени (вложенной энергии) временной интервал между пиками сигналов от С- и Н-компонентов $\Delta \tau_{\mathrm{H}-\mathrm{C}}$ уменьшается, что для заданного временно́го разрешения спектрометра (прежде всего ФЭУ) накладывает ограничение на верхний предел регистрируемых флюенсов ионов. Нижний предел в основном задается условием вложения в слой поверхностных загрязнений энергий ионов, достаточных для абляции этого слоя, например не ниже $10^{13} \mathrm{~cm}^{-2}$ за импульс для $300-400 \mathrm{keV}$ протонов, как было указано выше.

Самый первый импульс серии генерирует наивысший по амплитуде световой сигнал и флюенс ионов при максимальном измеренном с помощью калориметрии вложении энергии в титановую фольгу $(Q=85 \mathrm{cal})$. Поскольку перед самым первым импульсом любой серии поверхностные загрязнения, в том числе и электродов диода, максимальны, этот результат свидетельствует в пользу высокого вклада поверхностных загрязнений в эмиссию ионов электродной системой ускорителя. Второй импульс серии подтвердил этот вывод, поскольку был произведен через 2-3 s после первого, когда пауза между импульсами была редуцирована вдвое по сравнению с обычной в 4-5s. Это было сделано для того, чтобы оценить характерное время, необходимое для восстановления слоя поверхностных загрязнений.

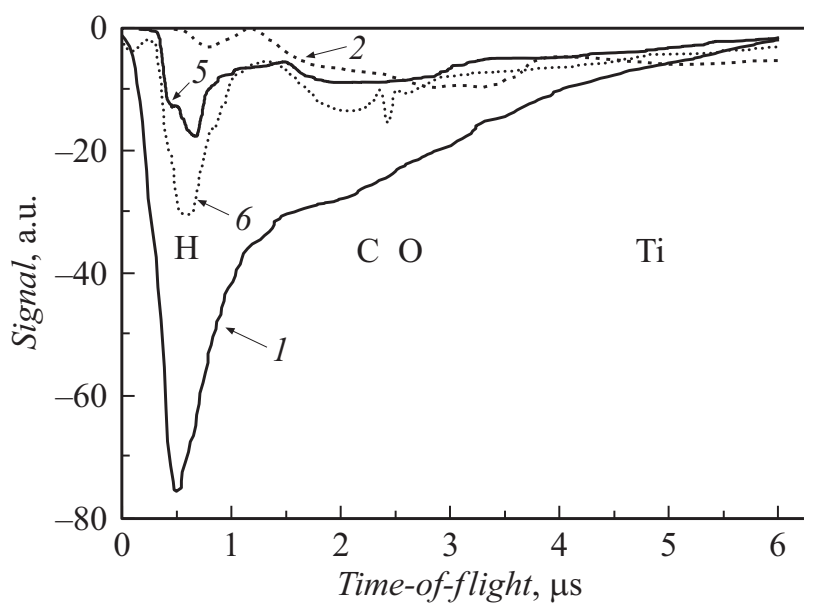

Рис. 2. Оптические времяпролетные спектры компонентов абляционной плазмы для разных импульсов серии. 
Суммарные результаты для четырех импульсов серии

\begin{tabular}{c|c|c|c|c|c|c|c|c|c|c|c|c|c|c}
\hline Номер импульса & Сила тока, А & $Q, \mathrm{cal}$ & $\tau_{\mathrm{H}}, \mu \mathrm{s}$ & $\tau_{\mathrm{C}}, \mu \mathrm{s}$ & $\tau_{\mathrm{H}} / \tau_{\mathrm{C}}$ & $\Delta \tau_{\mathrm{C}-\mathrm{H}, \mu \mathrm{s}}$ & $Q^{*}, \mathrm{cal}$ & $\delta, \%$ & $Q^{* *}, \mathrm{cal}$ & $\tau_{\mathrm{O}, \mu \mathrm{s}}$ & $\tau_{\mathrm{H}} / \tau_{\mathrm{O}}$ & $\tau_{\mathrm{Ti}}, \mu \mathrm{s}$ & $\tau_{\mathrm{H}} / \tau_{\mathrm{Ti}}$ \\
\hline 1 & 19 & 80 & 0.56 & 1.87 & 3.34 & 1.31 & 80 & - & 75.3 & - & - & - & - \\
2 & 12 & 35 & 0.8 & 2.77 & 3.46 & 1.97 & 35.4 & +1.1 & 33.3 & 3.44 & 4.3 & 5.5 & 6.88 \\
5 & 14 & 65 & 0.61 & 2.02 & 3.31 & 1.41 & 69 & +6.2 & 65 & - & - & 4.34 & 7.11 \\
6 & 11 & 50 & 0.66 & 2.23 & 3.38 & 1.57 & 55.7 & +11.4 & 52.4 & 2.4 & 3.63 & 4.2 & 6.36
\end{tabular}

Во втором импульсе не только снизилась амплитуда светового сигнала (в 10 раз), но и увеличилась разница времен пролета между пиками водородного и углеродного компонентов абляционной плазмы, что указывает на снижение ее температуры и, как следствие, на резкое снижение вложенной ионами энергии - за счет снижения их флюенса. Возвращение к нормальной частоте следования импульсов в 4-5s, найденной для ускорителя ТЕМП-1 эмпирически [1], позволило выйти на уровень вложений тепловой энергии в титановую фольгу в пределах 50-65 cal, что в среднем примерно в 1.4 раза ниже, чем в самом мощном (первом) импульсе, но в 1.7 раза выше, чем в искусственно ,Подавленном“ втором.

Колонка значений $Q^{*}$ дает представление о вложенных энергиях, если их рассчитывать по разнице времен пролета базы длиной $L=2 \mathrm{~cm}$ ионами углерода и водорода $\Delta \tau_{\mathrm{C}-\mathrm{H}}$, калибруя последующие значения серии импульсов по калориметрическим данным для самого первого импульса, т. е. принимая $Q_{1}^{*}=80 \mathrm{cal}$. Отличие от данных колонки $Q$, выраженное в процентах в колонке $\delta=100 \%\left(Q-Q^{*}\right) / Q$, дает представление о погрешности подобного определения вложенной энергии или флюенсов протонов, в среднем завышающего значения в последующих импульсах (относительно первого) примерно на $+6.2 \%$ (со среднеквадратичным отклонением $\pm 5.9 \%)$, что, вероятно, обусловлено отмеченной специфичностью самого первого импульса любой серии как самого мощного и, скорее всего, включающего максимальную примесь тяжелых ионов (преимущественно углерода). Выбор в качестве калибрующего значения $Q^{* *}=65 \mathrm{cal}$ для пятого („обычного“) импульса серии обнаруживает снижение погрешности определения вложенной энергии (и флюенса протонов) - в среднем до минус $2 \%$ (с тем же среднеквадратичным отклонением $\pm 5.9 \%$ ).

Относительное положение всех пиков тоже хорошо согласуется с предположением о их происхождении как об аблировавших компонентах поверхностного загрязнения - в основном $\mathrm{H}, \mathrm{C}$ и $\mathrm{O}$, а также частично поверхности самой титановой матрицы. Согласно исходным предположениям с грубыми упрощениями, отношения времен пролета отдельными компонентами должны быть следующими:

$\tau_{\mathrm{H}} / \tau_{\mathrm{C}}=\sqrt{12 / 1}=3.463$, что соответствует обнаруженному среднему для четырех импульсов серии $(3.37 \pm 0.07)$;
$\tau_{\mathrm{H}} / \tau_{\mathrm{O}}=\sqrt{16 / 1}=4$, что соответствует обнаруженному среднему $(3.96 \pm 0.5)$;

$\tau_{\mathrm{H}} / \tau_{\mathrm{Ti}}=\sqrt{47.9 / 1}=6.92$, что соответствует обнаруженному среднему $(6.78 \pm 0.4)$.

Если данные калориметрии находятся в согласии с времяпролетными данными, то результаты измерений электрического тока расходятся и с времяпролетными, и с данными калориметрии, что указывает на их ненадежность. В целом оптическая времяпролетная спектрометрия, сопряженная с калориметрическим измерением вложенной тепловой энергии, обнаруживает хорошую воспроизводимость результатов на уровне не хуже $\pm 6 \%$. Обнаруженные различия данных по вложенной тепловой энергии (флюенсам протонов) для первого и второго (искусственно подавленного) импульсов, а также для последующих импульсов серии указывают на то, что поверхностные загрязнения могут быть доминирующим источником ионов для электродов, работающих на принципе взрывной эмиссии, что открывает дополнительные возможности, в том числе и для коллективного ускорения ионов.

\section{Финансирование работы}

Работа выполнена при финансовой поддержке гранта Российского научного фонда (грант 17-19-01442).

\section{Конфликт интересов}

Авторы заявляют, что у них нет конфликта интересов.

\section{Список литературы}

[1] Remnev G.E., Isakov I.F., Opekunov M.S., Matvienko V.M., Ryzhkov V.A., Struts V.K., Grushin I.I., Zakoutayev A.N., Potyomkin A.V., Tarbokov V.A., Pushkaryov A.N., Kutuzov V.L., Ovsyannikov M.Yu. // Surface Coat. Technol. 1999. V. 114. P. 206-212.

[2] Аруев Н.Н., Козловский М.А., Кудояров М.Ф., Патрова М.Я., Романов П.А., Тюкальцев Р.В., Федичкин И.Л., Филиппов С.В. // Письма в ЖТФ. 2019. Т. 45. В. 16. C. $30-32$.

[3] Новодворский О.А., Михалевский В.А., Гусев Д.С., Лотин А.А., Паршина Л.С., Храмова О.Д., Черебыло Е.А. // Письма в ЖТФ. 2018. Т. 44. В. 6. С. 103-110.

[4] Zhang J., Tan C., Wang W., Wang Y., Ma T. // J. Korean Phys. Soc. 2003. V. 42. P. 885-889. 